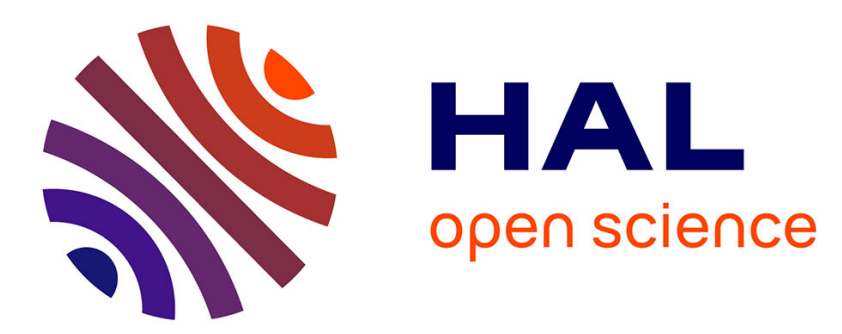

\title{
Decentralized handovers in cellular networks with cognitive terminals
}

\author{
Elena Veronica Belmega, Samson Lasaulce, Merouane Debbah
}

\section{To cite this version:}

Elena Veronica Belmega, Samson Lasaulce, Merouane Debbah. Decentralized handovers in cellular networks with cognitive terminals. ISCCSP-2008, Mar 2008, Malta. 6 p. hal-00328172v2

\section{HAL Id: hal-00328172}

\section{https://hal-centralesupelec.archives-ouvertes.fr/hal-00328172v2}

Submitted on 9 Feb 2009

HAL is a multi-disciplinary open access archive for the deposit and dissemination of scientific research documents, whether they are published or not. The documents may come from teaching and research institutions in France or abroad, or from public or private research centers.
L'archive ouverte pluridisciplinaire HAL, est destinée au dépôt et à la diffusion de documents scientifiques de niveau recherche, publiés ou non, émanant des établissements d'enseignement et de recherche français ou étrangers, des laboratoires publics ou privés. 


\section{Decentralized handovers in cellular networks with cognitive terminals \\ (Invited paper for the Session on MIMO Systems)}

\author{
Elena Veronica Belmega \\ Laboratoire des Signaux et Systèmes \\ CNRS - Supélec - Univ. Paris Sud \\ 91191 Gif-sur-Yvette, France \\ belmega@1ss.supelec.fr
}

\author{
Samson Lasaulce \\ Laboratoire des Signaux et Systèmes \\ CNRS - Supélec - Univ. Paris Sud \\ 91191 Gif-sur-Yvette, France \\ lasaulce@lss.supelec.fr
}

\author{
Mérouane Debbah \\ Chaire Alcatel - Lucent \\ Supélec \\ 91191 Gif-sur-Yvette, France \\ merouane.debbah@supelec.fr
}

\begin{abstract}
We consider a network that comprises a group of $K$ users, equipped with single-antenna terminals, who want to selfishly maximize their individual transmission rates and $S$ single-antenna base stations. The information rates and the transmit power levels of the users are not dictated by the base stations. In this context we introduce the problem of decentralized handovers: each terminal is equipped with a cognitive radio used to sense the quality of its links with the different base stations and share, in a smart way, its transmit power among them. In the hard handover case, for which there is a unique stable Nash equilibrium, we determine the selfish repartition of the users between the base stations and then we assess its social efficiency, which is measured in terms of sum-rate. In the soft handover case, the problem consists in determining the optimum selfish power allocation for each user and analyzing its sum-rate efficiency with respect to the equivalent virtual $K \times S$ multiple input multiple output (MIMO) network. We also show how to extend the provided results to the case of multi-antenna terminals and base stations and provide simulations to illustrate the presented results.
\end{abstract}

\section{INTRODUCTION}

The concept of decentralized or distributed wireless networks and the underlying problem of resource allocation has been properly formalized for the first time by Goodman et al. in [1] and [2]. The most simple decentralized channel is the multiple access channel (MAC), which allows one to describe the downlink of a cellular system with one base station (BS) and several mobile stations (MS). The MAC is said to be decentralized in the sense that a base station does not dictate to the users connected to it, their information rates and transmit power levels. Each user can choose freely its power allocation policy in order to selfishly maximize a certain individual performance criterion. In this paper we consider a system of $S$ orthogonal MACs. More precisely, the system comprises $S$ base stations using non-overlapping bands of frequency and a group of $K$ mobile stations. We also assume that the base stations are connected through perfect communication links. In UMTS networks for example, the base stations are connected through a radio network controller and very reliable wire connections (e.g. optic fibers), which are not far from perfect communication links. The system under investigation corresponds to the decentralized counterpart of the system analyzed by [3][4] where the authors assessed the benefits of cross-system diversity.

In this context we introduce and investigate the problem of decentralized handover in cognitive networks i.e. we want to know how free and selfish users would connect to the different base stations. Each user is assumed to be interested in maximizing its own transmission rate in the sense of Shannon. If hard handover is imposed to the system, the task of a given user is to decide which is the best base station for him to be connected to, given the fact the BSs have different characteristics. In this paper they differ by their reception noise levels and numbers of served users (which correspond to various multi-user interference levels) but they might also differ by their numbers of antennas, bandwidths or any other parameter likely to determine the transmission rate. On the other hand, if soft handover is allowed, the task of a user consists in optimally sharing its transmit power between the base stations in presence. The reason why we assume the mobile terminals to be equipped with a cognitive radio [5] is that we want the users to be as independent of the base stations as possible. Thanks to its cognitive radio each user can sense its environment, evaluate the transmission rate associated with a given route, and make the optimal decision in the sense of his utility. Therefore the problem addressed in this paper can be seen as a generalized version of the "car versus public transportation" problem analyzed in [6] to the context of wireless networks. In [6] the authors considered commuters who want go from Berkeley to San Francisco. Commuters can either drive over the Bay Bridge or take the BART (bay area rapid transit) train. Each commuter acts selfishly and chooses the route that minimizes his own transportation time. Assuming reasonable functions for commuting time versus number of users for the two routes, it is possible to show the existence and uniqueness of a stable Nash equilibrium (NE) for this game [6]. In our setup this situation corresponds to a network with two base stations for which only hard handover is allowed and a crowded route corresponds to a base station with a high multiuser interference level.

This paper is structured as follows. In the first main part (Sec. II) single-antenna terminals and Gaussian channels are assumed. Both the hard and soft handover cases are treated. For each of these cases the network sum-rate achieved by 
using single-user decoding (SUD) or successive interference cancelation (SIC) is evaluated and compared to the sum-rate of the centralized counterpart of the system under investigation (the latter corresponds to the sum-rate of the equivalent virtual MIMO network), which allows us to measure the social efficiency of the considered networks of selfish users. The second main part (Sec. III) is intended to show to extend the provided results to the case of fading channels with multiantenna terminals. Because of this and the lack of space, only one scenario with multi-antenna terminals is detailed: the hard handover case with single-user decoding.

\section{NETWORKS OF SINGLE-ANTENNA TERMINALS WITH STATIC LINKS}

\section{A. Signal model}

The global system under investigation is represented in Fig. 1. It consists of $K$ mobile stations and $S$ base stations using non-overlapping bands of frequency (in Fig. 1, $S=3$ ) which are taken to be equal and normalized at $1 \mathrm{~Hz}$. All terminals have only one antenna. The equivalent baseband signals received by base station $s \in\{1, \ldots, S\}$ can be written as

$$
Y_{s}=\sum_{k=1}^{K} h_{s, k} X_{s, k}+Z_{s}
$$

where $\forall k \in\{1, \ldots, K\}, \forall s \in\{1, \ldots, S\}, X_{s, k}$ is the signal transmitted by user $k$ to base station $s, h_{s, k}$ is the gain of the corresponding channel, which is assumed to be constant in this section, and $Z_{s}$ is a complex white Gaussian noise distributed as $\mathcal{N}\left(0, N_{s}\right)$. Each user has a limited transmit power: $\mathbb{E}\left|X_{s, k}\right|^{2}=\alpha_{s, k} P$ with $\sum_{s=1}^{S} \alpha_{s, k}=1$ and $\alpha_{s, k} \in\{0,1\}$ for the hard handover case and $\alpha_{s k} \in[0,1]$ for the soft handover case. We assume that the mobile terminals have the same transmit power, which is a reasonable assumption in most wireless systems.

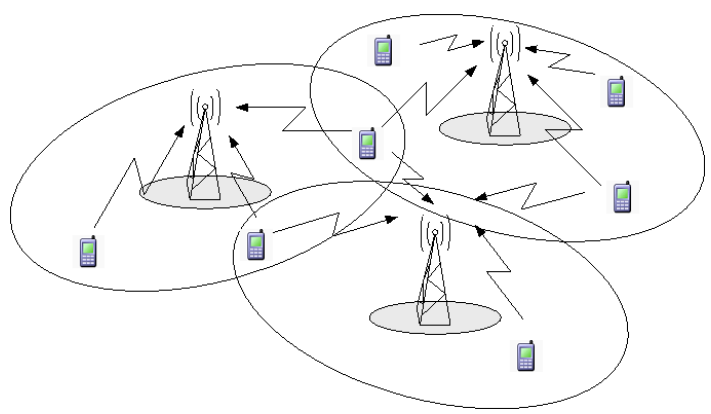

Fig. 1. A group of $K$ free, selfish and cognitive users who want to connect to one (hard handover case) or several (soft handover case) base stations having non-overlapping bands of frequency.

At the receivers, we assume that the same decoding scheme is used: either all base stations implement single-user decoding or successive interference cancelation. The first decoding scheme can be assumed, for instance, because the base station has to be neutral in the game or/and for limiting the receiver complexity. The second decoding scheme is more efficient in terms of interference reduction but is intrinsically unfair. In order for this decoding scheme to be fair from we will introduce a coordination mechanism that allow each user to see all decoding ranks instead of a single one.

\section{B. Hard handover case}

In this section the users can connect to only one base station at a time. Each user chooses the best base station in the sense of his utility function which is chosen to be the transmission rate (the latter depends on the decoding scheme used at the base stations). For this purpose each user knows the reception noise level at each base station $s$ and is able to sense its multiuser interference level. In this section we will assume for simplicity that the channel gains $h_{s k}=1$ for all $(s, k)$. Let $K_{s}$ be the number of users in system $s \in\{1, \ldots, S\}$, and $x_{s}=\frac{K_{s}}{K}$, the fraction of the users in system $s$, with $\sum_{s=1}^{S} x_{s}=1$. By definition $x_{s} \in \mathbb{Q}^{+}$but for sufficiently high numbers of users it is reasonable to assume that $x_{s} \in \mathbb{R}^{+}$. Note that if there was no interference all the users would connect to the base station $s_{\min }$ with $N_{s_{\text {min }}}=\min \left\{N_{s}\right\}$ and therefore $x_{s}=1$ for $s=s_{\min }$ and $x_{s}=0$ otherwise. Because of the multiuser interference the users will choose another repartition in general. The main issue we want to solve now is to determine this repartition and evaluate its network sum-rate efficiency.

1) Single-user decoding: By assumption $\alpha_{s k} \in\{0,1\}$. Therefore the utility for user $k$ in system $s$ merely writes as:

$$
u_{k}^{(s)}=\log _{2}\left[1+\frac{P}{N_{s}+\left(K x_{s}-1\right) P}\right] \text {. }
$$

As the users are cognitive, they can sense their environment and know what are their utilities at any time. If we consider the dynamic game where the cognitive users have to choose between the two base stations. It can be shown [6] that the users are going to react as long as their utility in system $s$ is not equal to that in system $j$ where $j \in\{1, \ldots, S\}$. This observation - reaction process is going to converge to a stable equilibrium point given by:

$\log _{2}\left[1+\frac{P}{N_{s}+\left(K x_{s}-1\right) P}\right]=\log _{2}\left[1+\frac{P}{N_{j}+\left(K x_{j}-1\right) P}\right]$

Knowing also that $\sum_{j=1}^{S} x_{j}=1$ we obtain the repartition of the selfish users:

$$
x_{s}^{*, S U D}=\frac{1}{S}+\frac{1}{S} \sum_{j=1}^{S} \frac{N_{j}-N_{s}}{K P} .
$$

Furthermore, the solution $x_{s}^{*}$ has to lie in $[0,1]$, for all $s \in\{1, \ldots S\}$. In order to find the optimal distribution that satisfies these constraints we propose the following recursive algorithm:

1) Initialization: $\mathcal{S}=\{1, \ldots S\}$ 
2) For all the BSs $s \in \mathcal{S}$ apply equation (4) and find $x_{s}^{*}$;

3) If there exists a non empty subset $\mathcal{S}_{0} \subset \mathcal{S}$ such that $x_{s}^{*}<0$ for all $s \in \mathcal{S}_{0}$ :

a) $x_{s}^{*}=0$ for all $s \in \mathcal{S}_{0}$;

b) Update: $\mathcal{S}=\mathcal{S} \backslash \mathcal{S}_{0}$ and $S=|\mathcal{S}|$;

c) Return to step 2;

4) If there exists a non empty subset $\mathcal{S}_{1} \subset \mathcal{S}$ such that $x_{s}^{*}>1$ for all $s \in \mathcal{S}_{1}$ :

a) $x_{s}^{*}=0$ for all $s \in \mathcal{S} \backslash \mathcal{S}_{1}$;

b) Update: $\mathcal{S}=\mathcal{S}_{1}$ and $S=|\mathcal{S}|$

c) Return to step 2 .

where $|\mathcal{S}|$ denotes the cardinal of the set $\mathcal{S}$. Due to space limitations we will not prove the existence and uniqueness of the mentioned Nash equilibrium but this can be done by calling Theorems 1 and 2 of [8].

Finally, the network sum-rate can be evaluated and is equal to:

$R_{\text {sum }}^{*, S U D}=\sum_{s=1}^{S} K x_{s}^{*, S U D} \log _{2}\left[1+\frac{P}{N_{s}+\left(K x_{s}^{*, S U D}-1\right) P}\right]$

2) Successive interference cancellation: When successive interference cancellation is assumed, the base station $s$ has to decide in which order it decodes the $K_{s}$ users. If these choices are made once and for all, the game is unfair since the users do not see the same amount of interference for a given base station. In order to make the game fair we propose to use the coordination mechanism introduced in [7] for MIMO multiple access channels. Each base station $s$ owns a random source that generates a discrete signal with $K_{s}$ ! equiprobable states. The realization of this random signal precisely indicates in which order the users connected to the base station $s$ are decoded using a perfect successive interference canceller. The base station discloses the realizations of this signal to all the users. The frequency to which the realizations are drawn can be chosen such that a certain degree of fairness is reached over the duration of the transmission. In a real wireless system it could be chosen to be roughly proportional to the inverse of the channel coherence time. The utility for user $k$ if it connects to $\mathrm{BS} s$ is therefore:

$$
u_{k}^{(s)}=\sum_{i=1}^{K_{s}} p_{k}^{(s)}(i) \log _{2}\left[1+\frac{P}{N_{s}+(i-1) P}\right]
$$

where $p_{k}^{(s)}(i)$ is the probability that user $k$ is decoded with rank $i$ in system $s$. Knowing that the BS use a fair SIC we have that $p_{k}^{(s)}(i)=\frac{1}{K_{s}}$ for all $i \in\left\{1, \ldots, K_{s}\right\}$ and the utility function becomes:

$$
u_{k}^{(s)}=\frac{1}{K x_{s}} \log _{2}\left[1+\frac{K x_{s} P}{N_{s}}\right] .
$$

At the equilibrium we have that for a fixed $s \in\{1, \ldots, S\}$, $\forall j \in\{1, \ldots, S\}:$

$$
\frac{1}{x_{s}} \log _{2}\left[1+\frac{K x_{s} P}{N_{s}}\right]=\frac{1}{x_{j}} \log _{2}\left[1+\frac{K x_{j} P}{N_{j}}\right]
$$

While the corresponding system of equations can be solved by a simple numerical technique for $S=2$, the problem is more challenging for $S>2$, for which the solution goes the scope of this paper. At the equilibrium point, the sum rate obtained in this case is:

$$
R_{\text {sum }}^{*, S I C}=\sum_{s=1}^{S} \log _{2}\left[1+\frac{K x_{s}^{*, S I C} P}{N_{s}}\right] .
$$

3) Full cooperation upper bound: Here we consider the network to be centralized and express its sum-rate, which correspond to the transmission rate of the equivalent $K \times S$ virtual MIMO channel since the considered network consists of $S$ parallel (or orthogonal) MACs. For a given choice for the fractions $x_{1}, \ldots, x_{S}$ the network sum-rate writes:

$$
R_{\text {sum }}^{C}=\sum_{s=1}^{S} \log _{2}\left[1+\frac{K x_{s} P}{N_{s}}\right] .
$$

Although the coordination mechanism proposed for the case of SIC is a priori suboptimal, we observe that the sum-rate function for centralized case $R_{\text {sum }}^{C}\left(x_{s}\right)$ is exactly the same as that for the decentralized case with SIC $R_{\text {sum }}^{S I C}\left(x_{s}\right)$ but the selfish behavior of the users will not lead, in general, to the repartition maximizing this function $R_{\text {sum }}^{S I C} \equiv R_{\text {sum }}^{C}$. However, this observation shows the interest in using a pricing mechanism to deviate the users from the spontaneous Nash equilibrium into a stimulated one.

The optimal centralized network sum-rate when hard handover is imposed is obtained by maximizing the following Lagrangian:

$$
\mathcal{L}=-R_{\text {sum }}^{C}+\lambda\left(\sum_{s=1}^{S} x_{s}-K\right) .
$$

By setting the derivative $\frac{\partial \mathcal{L}}{\partial x_{j}}$ to zero for all $j \in\{1, \ldots S\}$, we obtain the following water-filling solution:

$$
x_{j}^{*, C}=\frac{1}{K}\left[\frac{1}{\lambda}-\frac{N_{j}}{P}\right]^{+}
$$

where the parameter $\lambda \geq 0$ is tuned such that the power constraints are met at the optimal point: $\sum_{s=1}^{S} x_{s}^{*, C}=1$. It can be checked that this repartition of users (eq. (12)) is the same as for the decentralized network with single-user decoding (eq. (4)).

\section{Soft handover case}

In this section $\alpha_{s, k}$ is not imposed to belong to a binary set (hard handover) but lies in $[0,1]$. Also we assume from now on that the channel gains $h_{s, k}$ can be arbitrary.

1) Single-user decoding: The utility (transmission rate) for user $k$ expresses now as:

$$
u_{k}=\sum_{s=1}^{S} \log _{2}\left[1+\frac{\alpha_{s, k} \eta_{s k}}{1+\sum_{\ell \neq k} \alpha_{s, \ell} \eta_{s \ell}}\right]
$$


with $\eta_{s, k}=\frac{\left|h_{s, k}\right|^{2} P}{N_{s}}$. We introduce the constrained functions:

$$
\mathcal{L}_{k}=-u_{k}+\lambda_{k}\left(\sum_{s=1}^{S} \alpha_{s, k}-1\right) .
$$

The optimal vector of (non-negative) fractions $\left\{\alpha_{s, k}\right\}_{s=1}^{S}$ is the solution of

$$
\left\{\frac{\partial \mathcal{L}_{k}}{\partial \alpha_{s, k}}=0 \quad, \forall k \in\{1, \ldots, K\}, s \in\{1, \ldots, S\} .\right.
$$

The parameters $\lambda_{k} \geq 0$ are tuned such that $\sum_{s=1}^{S} \alpha_{s, k}^{*, S U D}=1$. In order to better understand the nature of the problem to be solved let us detail the case $S=2, K=2$. The optimal pair of power fractions is the solution of the following system (KT optimality conditions):

$$
\left\{\begin{array}{ccc}
\frac{\eta_{11}}{1+\eta_{12}\left(1-\alpha_{2}\right)+\eta_{11} \alpha_{1}}-\frac{\eta_{21}}{1+\eta_{22} \alpha_{2}+\eta_{22}\left(1-\alpha_{1}\right)}+\lambda_{1}-\lambda_{2} & =0 \\
-\frac{\eta_{22}}{1+\eta_{12}\left(1-\alpha_{2}\right)+\eta_{11} \alpha_{1}}+\frac{1+\eta_{22} \alpha_{2}+\eta_{21}\left(1-\alpha_{1}\right)}{1}+\lambda_{3}-\lambda_{4} & =0 \\
\lambda_{1}\left(\alpha_{1}-1\right) & =0 \\
\lambda_{2} \alpha_{1} & =0 \\
\lambda_{3}\left(\alpha_{2}-1\right) & =0 \\
\lambda_{4} \alpha_{2} & =0 .
\end{array}\right.
$$

Now we consider a particular regime for the channel gains to show that the solution obtained is not always intuitive. Assume that for $\lambda_{1}=0, \lambda_{2}=0, \lambda_{3}=0$ and $\lambda_{4}=0$, the solution of the previous system $\left(\alpha_{1}^{*}, \alpha_{2}^{*}\right)$ is such that $\alpha_{1}^{*} \in[0,1]$ and therefore that the solution expresses as:

$$
\left\{\begin{aligned}
\alpha_{1}^{*} & =\frac{\eta_{12}+\eta_{22}+\eta_{12} \eta_{21}+\eta_{12} \eta_{22}}{\eta_{11} \eta_{22}-\eta_{12} \eta_{21}} \\
\alpha_{2}^{*} & =\frac{\eta_{11}+\eta_{21}+\eta_{11} \eta_{21}+\eta_{12} \eta_{21}}{\eta_{11} \eta_{22}-\eta_{12} \eta_{21}}
\end{aligned}\right.
$$

We see that this solution is not that easy to interpret in general. Let us focus on the special case where user 1 has a dominant link that is $\eta_{11}>>\eta_{21}$. Then

$$
\begin{aligned}
\alpha_{1}^{*} & =\frac{\eta_{12}+\eta_{22}+\eta_{12} \eta_{21}+\eta_{12} \eta_{22}}{\eta_{11}\left(\eta_{22}-\eta_{12} \frac{\eta_{21}}{\eta_{11}}\right)} \\
& \simeq \frac{1}{\eta_{11}}\left(1+\eta_{12}+\frac{\eta_{12}}{\eta_{22}}\right)+\frac{\eta_{21}}{\eta_{11}} \frac{\eta_{12}}{\eta_{22}} \\
& \simeq \frac{1}{\eta_{11}}\left(1+\eta_{12}+\frac{\eta_{12}}{\eta_{22}}\right) .
\end{aligned}
$$

As a consequence if the link 11 is strong user 1 will allocate a small fraction of his power to it and save it to allocate almost all his power to the weak link. This situation is the contrary to what happens with a water-filling type power allocation policy. In fact user 1 will allocate more power to link 11 only when user 2 generates a large amount of interference in its strongest link. When $\eta_{12}>>1$ we have

$$
\alpha_{1}^{*} \simeq \min \left\{\frac{\eta_{12}}{\eta_{11}}, 1\right\} .
$$

Thus for user 1 the fraction of power in system 1 is simply the ratio of the interference level to the useful signal level.
2) Successive interference cancellation: We assume that the base stations use the same fair SIC decoding technique as described for the hard handover case. The only difference here is that all the users can be connected to all the base stations $S$ at the same time. The utility for user $k$ is:

$u_{k}=\sum_{s=1}^{S} \frac{1}{K} \sum_{i=1}^{K}\left[\frac{1}{\left(\begin{array}{c}K-1 \\ i-1\end{array}\right)} \sum_{J_{k}^{(i-1)}} \log _{2}\left(1+\frac{\eta_{s k} \alpha_{k}(s)}{1+\sum_{l \in J_{k}^{(i-1)}} \eta_{s l} \alpha_{l}(s)}\right)\right]$

where $J_{k}^{(i-1)}=\{I \subset\{1, \ldots, K\}-\{k\}$, such that: $|I|=i-1\}$. We introduce the Lagrange function:

$$
\mathcal{L}_{k}=-u_{k}+\lambda_{k}\left(\sum_{s=1}^{S} \alpha_{k}(s)-1\right)
$$

The equilibrium in this case is the solution of:

$$
\left\{\frac{\partial \mathcal{L}_{k}}{\partial \alpha_{k}(s)}=0 \quad \forall k \in\{1, \ldots, K\}, s \in\{1, \ldots, S\}\right.
$$

The parameters $\lambda_{k} \geq 0$ are tuned such that $\sum_{s=1}^{S} \alpha_{k}^{*, S I C}=1$. Always for the purpose of identifying the nature of the mathematical problem to be solved we restrict our attention to the case where $S=2$ and $K_{*} \overline{\overline{S I}} C$. In this case the optimal allocation point $\left(\alpha_{1}^{*, S I C}, \alpha_{2}^{*, S I C}\right)$ is the solution to the following system:

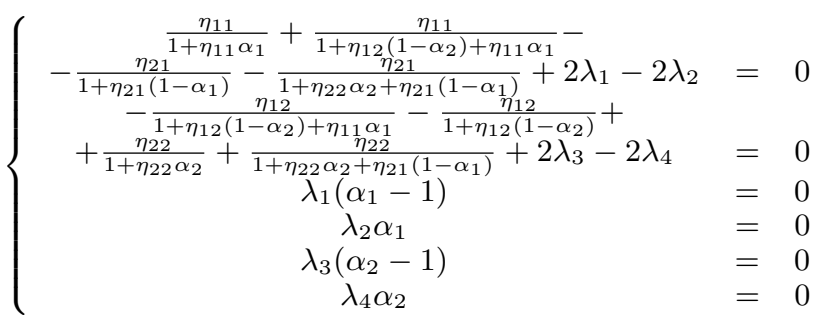

3) Full cooperation upper bound: For an arbitrary number of users in system s, $K_{s}$ the network sum-rate writes:

$$
R_{\text {sum }}^{C}=\sum_{s=1}^{S} \log _{2}\left[1+\sum_{k=1}^{K_{s}} \eta_{s k} \alpha_{s, k}\right],
$$

which is optimized by introducing the following constrained sum-rate function:

$$
\mathcal{L}=-R_{\text {sum }}^{C}+\lambda_{k}\left(\sum_{s=1}^{S} \alpha_{s, k}-1\right) .
$$

Like the hard handover case two observations can be made: $R_{\text {sum }}^{S I C}\left(\alpha_{s, k}\right)=R_{\text {sum }}^{C}\left(\alpha_{s, k}\right)$ and $\alpha_{s, k}^{*, C}=\alpha_{s, k}^{*, S U D}$. The first observation always indicates the potential of a pricing mechanism in our context. Simulations will help us to assess the gap between the performance of the centralized and decentralized networks and quantify this potential. The second observation shows that the selfish power allocation when SUD is assumed at the base stations is the same as in a cooperative network with an optimal decoding scheme. 


\section{HARD HANDOVER IN NETWORKS OF MULTI-ANTENNA TERMINALS WITH FADING LINKS}

In the previous section we have assumed static channels and single-antenna terminals. Our approach can be extended to the more attractive scenario where fading channels and multiple antennas are assumed. Because of space limitation we will only detail one case, which is the hard handover case with single-user decoding. The equivalent baseband signal received by BS $s \in\{1, \ldots, S\}$ is now given by:

$$
\underline{Y}_{s}(\tau)=\sum_{k=1}^{K} \mathbf{H}_{s, k} \underline{X}_{s, k}(\tau)+\underline{Z}_{s}(\tau)
$$

where $\forall k \in\{1, \ldots, K\}, \forall s \in\{1, \ldots, S\}, \underline{X}_{s, k}(\tau)$ is the signal transmitted by user $k$ to base station $s$ at time $\tau$, $\mathbf{H}_{s, k}(\tau) \in \mathbb{C}^{n_{r} \times n_{t}}$ is the channel matrix associated with user $k$ for the system $s$ (stationary and ergodic process), $n_{r}$ the number of receive antennas (the same for all base stations), $n_{t}$ the number of transmit antennas (the same for all users), $\underline{Z}_{s}(\tau)$ is an $n_{r}$-dimensional complex white Gaussian noise distributed as $\mathcal{N}\left(\underline{0}, n_{0} B_{s} \mathbf{I}\right)$, where $n_{0}$ is the receive noise power spectral density, $B_{s}$ the bandwidth of system $s$ and $\rho_{s}$ is the corresponding signal-to-noise ratio (SNR). We denote by $N_{s}=n_{0} B_{s}$. For simplicity we will omit the time index $\tau$ from our notations. Each user has a limited transmit power: $\sum_{s=1}^{S} \mathbb{E}\left|\underline{X}_{s, k}\right|^{2} \leq 1$. In our analysis the flat fading channel vectors of the different links can possibly vary from symbol vector (or space-time codeword) to symbol vector (or space-time codeword). We assume that the receivers (base stations) know their channel matrix (coherent communication assumption) and send the information through reliable links to a central controller.

For simplicity we assume that $S=2$ and also that there is neither transmit spatial correlation nor receive spatial correlation. The input covariance matrix for user $\mathrm{k}$ in system 1 is of the form $\mathbf{Q}_{1 k}=\alpha_{1 k} P \mathbf{I}$ with $\alpha_{k} \in\{0,1\}$. Also $\mathbf{Q}_{2 k}=\overline{\alpha_{k}} P \mathbf{I}$. Assuming the asymptotic regime in terms of the number of antennas: $n_{t} \longrightarrow \infty, n_{r} \longrightarrow \infty$, and

$\lim _{\rightarrow \infty, n_{r} \rightarrow \infty} \frac{n_{t}}{n_{r}}=c<\infty$ and by exploiting Theorem 3.7 of [9] we find that the approximated transmission rate per receive antenna for user $\mathrm{k}$ when he puts all his power in system 1 writes

$$
\begin{aligned}
u_{k}^{(1)}= & n_{r}\left[K x_{1} c \log _{2}\left(1+K x_{1} \rho_{1} P \gamma_{1 k}\right)-\log _{2}\left(K x_{1} c \gamma_{1 k}\right)+\right. \\
& +K x_{1} c \gamma_{1 k} \log _{2} e-K x_{1}^{\prime} c \log _{2}\left(1+K x_{1}^{\prime} \rho_{1} P \gamma_{1 k}^{\prime}\right)+ \\
& \left.+\log _{2}\left(K x_{1}^{\prime} c \gamma_{1 k}^{\prime}\right)-K x_{1}^{\prime} c \gamma_{1 k}^{\prime} \log _{2} e\right]
\end{aligned}
$$

with

$$
\begin{aligned}
& \left\{\begin{aligned}
\gamma_{1 k} & =\frac{1}{K x_{1} c} \frac{1}{1+K x_{1} \rho_{1} \delta_{1 k}} \\
\delta_{1 k} & =\frac{P}{1+K x_{1} \rho_{1} P \gamma_{1 k}},
\end{aligned}\right. \\
& \left\{\begin{array}{l}
\gamma_{1 k}^{\prime}=\frac{1}{K x_{1}^{\prime} c} \frac{1}{1+K x_{1}^{\prime} \rho_{1} \delta_{1 k}^{\prime}} \\
\delta_{1 k}^{\prime}=\frac{P}{1+K x_{1}^{\prime} \rho_{1} P \gamma_{1 k}^{\prime}}
\end{array}\right.
\end{aligned}
$$

and $x_{1}^{\prime}=x_{1}-\frac{1}{K}, \lim _{n_{t} \rightarrow \infty, n_{r} \rightarrow \infty} \frac{n_{t}}{n_{r}}=c<\infty, \rho_{1}=\frac{1}{N_{1}}$. As $\gamma_{1 k}$ and $\gamma_{1 k}^{\prime}$ are functions of $K_{1}$, the utility of user $\mathrm{k}$ in system 1 can be written as $u_{k}^{(1)}=\phi_{1}\left(K_{1}\right)$ and $u_{k}^{(2)}=\phi_{2}\left(K_{1}\right)$ in system 2. The determination of the repartition of the users can therefore be determined.

\section{Simulation RESUlTS}

For $S=2, N_{1}=1, N_{2}=10, P=10$ and $K=20$, Fig. 2 shows the network sum-rate as a function of the fraction of users $x_{1}$ in base station 1 when hard handover is assumed. The bottom curve corresponds to the scenario where single-user decoding is assumed, whereas the two top curves coincide and correspond to the centralized and SIC-based scenarios. For the SUD scheme the spontaneous user repartition in BS 1 is about 0.5 while it exists a better repartition which would provide a sum-rate close to $5 \mathrm{bit} / \mathrm{s}$ instead of $3 \mathrm{bit} / \mathrm{s}$, which shows the potential of a pricing mechanism when SUD is used at the base stations. On the other hand, when SIC is assumed the optimal selfish repartition is about 0.75 and the corresponding sum-rate is quite close to the optimum sum-rate obtained with $x_{1}=0.52$. Pricing is apparently less promising when SIC is implemented.

Now consider the case of soft handover. The simulation setup for Fig. 3 is: $S=2, K=2, N_{1}=N_{2}=1, h_{11}=1$, $h_{21}=2, h_{12}=4, h_{22}=3$. This figure represents the sumrate versus the MS transmit power. Interestingly the sum-rate achieved by a decentralized network using SIC is close to that of a centralized network, which has been confirmed in many other simulation setups. This is a good news in the sense that intelligence can be transferred from the base station to the mobile stations and it shows the potential for reducing the overhead due to the network signalling and control. This also shows that the sub-optimal fair coordination mechanism proposed is sum-rate efficient. Another observation we made is that, in some scenarios, a selfish network using SUD can perform better than a selfish network using SIC. This can be proved analytically. This happens when the following conditions are met: $-\frac{\eta_{11}}{1+\eta_{12}}+\frac{\eta_{21}}{1+\eta_{21}} \geq 0$ and $\frac{\eta_{12}}{1+\eta_{12}}-\frac{\eta_{22}}{1+\eta_{21}} \geq 0$. In this region of $\mathbb{R}^{4}$, the selfish users optimal power allocation when the BSs use SUD, is $\alpha_{1}^{*}=0$ and $\alpha_{2}^{*}=0$. The users use only one base station and their selfish behavior results in an interference-free system having a better sum-rate than that obtained by using SIC at the BSs.

At last, in Fig. 4, we have studied the case where mutiple antenna terminals are considered, and for which the channels are no longer static but time varying ones. The setup is: $S=2$, $K=20, N_{1}=1, N_{2}=10, n_{r}=n_{t}=4$. The decoding scheme used by the base stations single user decoding. The equilibrium corresponds to the crossing point between utility for user 1 and utility for user 2 . In the chosen setup the user repartition is quite uniform since $x_{1}^{*}=0.525$. This repartition occurs when the multi-user interference strongly dominates the noise. Then the BS reception noise level is no longer a discriminating characteristic for the base station. 


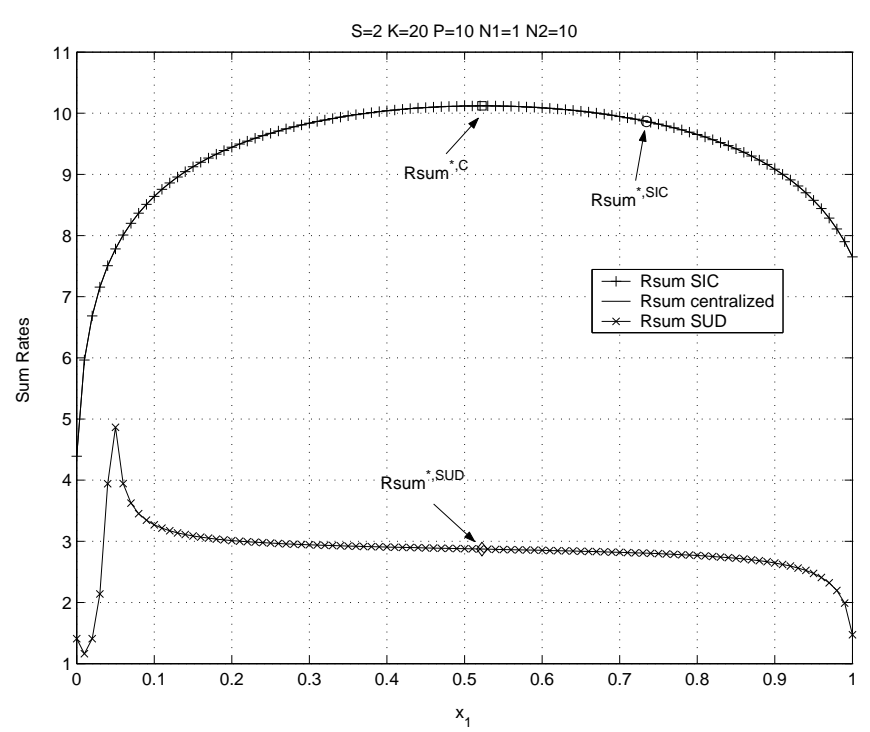

Fig. 2. Achievable sum rate versus user fraction in base station 1. Assumptions: hard handover, AWGN channels, single antenna terminals. Setup: $S=2, K=20, P=10, N_{1}=1, N_{2}=10$.

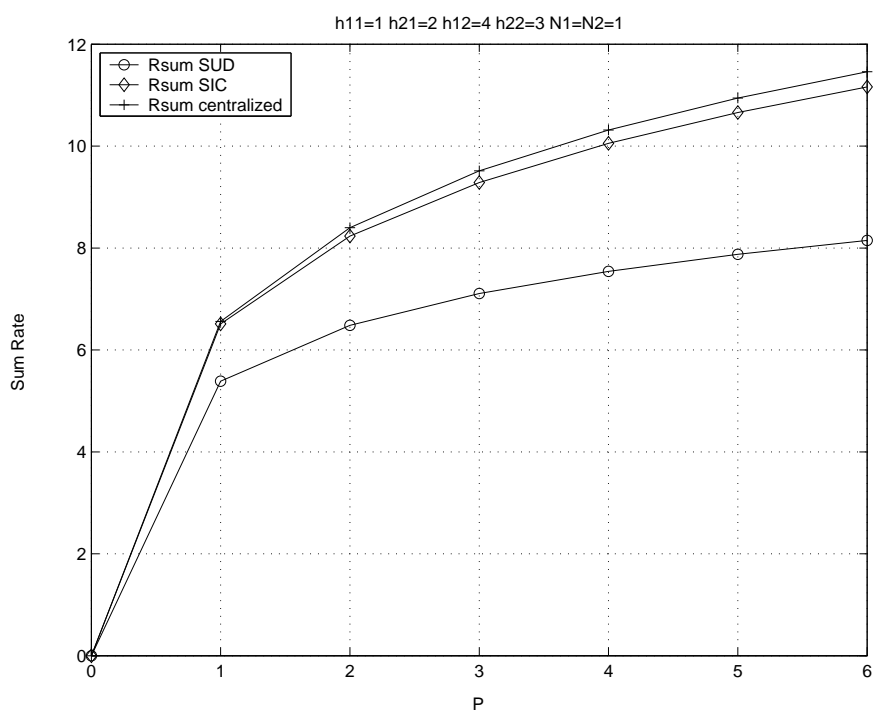

Fig. 3. Achievable sum rate versus MS transmit power. Assumptions: soft handover, AWGN channels, single antenna terminals. Setup: $S=2, K=2$, $N_{1}=N_{2}=1, h_{11}=1, h_{21}=2, h_{12}=4, h_{22}=3$.

\section{EXTENSIONS}

In the case of decentralized networks with cognitive terminals with hard handover the notion of preference has to be extended. In this paper a selfish user discriminates the base stations by their reception noise and multi-user interference levels. It would also be useful to analyze networks where BS have different bandwidths, numbers of antennas, spreading factors or any parameters influential on the user's utility (the transmission rate in this paper). We also saw the potential of devising a pricing mechanism in networks with hard handover, especially when single-user decoding is assumed at the

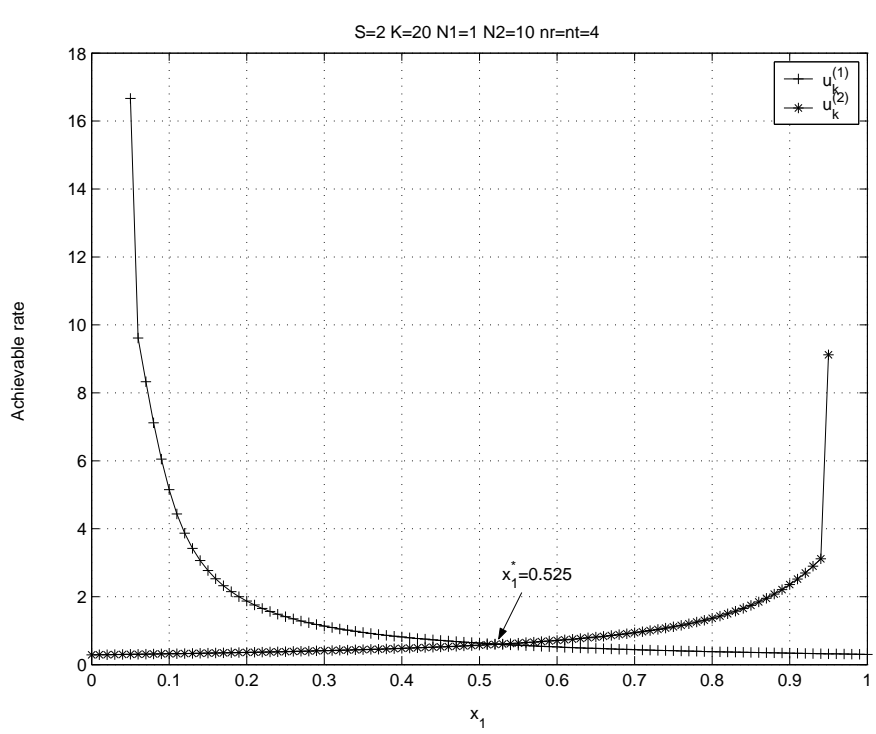

Fig. 4. User utilities versus user fraction in base station 1. Assumptions: hard handover, fading channels, multi-antenna terminals. Setup: $S=2, K=20$, $N_{1}=1, N_{2}=10, n_{r}=n_{t}=4$.

BSs. When SIC is assumed, the optimality of the proposed coordination mechanism should be assessed in order to know whether it is always sum-rate efficient. Also it is important to assess the performance improvement due to soft handover over hard handover in decentralized networks, which will be done in future work.

\section{REFERENCES}

[1] M. Serizawa and D. J. Goodman, “ Traffic sharing scheme for distributed dynamic channel allocation", in the IEE Proc. of the 7th European Conference on Mobile and Personal Communications, Dec. 1993, pp. 131-135.

[2] S. A. Grandhi, R. Vijayan and D. J. Goodman, "Distributed power control in cellular radio systems", IEEE Transactions on Communications, Vol. 42, No. 234, pp. 226-228, Feb/Mar/Apr 1994.

[3] S. Lasaulce, A. Suarez, R. De Lacerda and M. Debbah, "Cross-system resource allocation based on random matrix theory", in the ICST/ACM Proc. of the International Conference on Performance Evaluation Methodologies and Tools (Valuetools), Oct. 2007.

[4] S. Lasaulce, A. Suarez, R. De Lacerda and M. Debbah, "Using CrossSystem Diversity in Heterogeneous Networks: Throughput Optimization", Elsevier Journal of Performance Evaluation, submitted, Nov. 2007.

[5] B. A. Fette, "Cognitive Radio Technology", Newnes editors, 2006.

[6] A. K. Dixit and B. J. Nalebuff, "Thinking Strategically: The Competitive Edge in Business, Politics, and Everyday Life", Paperback, Chap. 9, pp. 228-231, 1993.

[7] S. Lasaulce, A. Suarez, M. Debbah and E. Altman, " Distributed power allocation for fast fading MIMO multiple access channels", IEEE Journal of Selected Areas in Communications (JSAC), special issue on Game Theory, submitted, Aug. 2007.

[8] J. Rosen, "Existence and uniqueness of equilibrium points for concave n-person games", Econometrica, Vol. 33, pp. 520-534, 1965.

[9] A. Tulino and S. Verdu, "Random Matrices and Wireless Communications", Foundations and trends in communications and information theory, NOW, The Essence of Knowledge, 2004. 